\title{
An Observational Investigation of Penetrative Convection
}

\author{
Niels Otto Jensen ${ }^{1}$ and D. H. Lenschow \\ National Center for Atmospheric Research, ${ }^{2}$ Boulder, CO 80309
}

(Manuscript received 27 March 1978, in final form 12 June 1978)

\begin{abstract}
Data taken during the Air Mass Transformation Experiment (AMTEX) by the NCAR Electra aircraft have proven useful for investigating the structure of thermals penetrating into the turbulent inversion layer which caps the convective mixed layer. On 16 February 1975, two flight legs, one upwind and one crosswind, and each about $12 \mathrm{~min}$ long ( $\sim 80 \mathrm{~km}$ in length), were flown at a nominally constant altitude at about the level of the turbulent inversion layer. Because of the variations in height of this relatively thin layer, the airplane spent about equal amounts of time above and below the inversion layer. These two legs are further split into six sections for statistical analyses, each with somewhat different characteristics. Variances, covariances, spectra and cospectra of potential temperature, the three air velocity components, and humidity are computed to illustrate the dynamic processes accurring in this region. Two spectral maxima occur in vertical velocity and temperature : one at a wavelength of about 1.5 times the mixed layer depth and the other at about $200-300 \mathrm{~m}$, which seems to be related to the characteristic size of a penetrating thermal.
\end{abstract}

\section{Introduction}

A large part of the heat and moisture transport in a convectively unstable atmospheric boundary layer (ABL) occurs in discrete convective elements which we call thermals. In the surface layer and in the lower part of the mixed layer they are encountered as ascending bursts of relatively warm and (usually) humid air. Their role in the dynamics of the $\mathrm{ABL}$ is qualitatively as follows: In rising through the mixed layer, most of their buoyancy is converted into vertical kinetic energy. As a thermal rises, however, turbulence is generated through shear at the interface with the ambient air, enhancing turbulent diffusion and resulting in a gradual dilution of the thermal's heat and moisture content. Eventually the thermal may impinge on the mixed-layer capping inversion, overshoot the mean height of this interface, become negatively buoyant and finally sink back-all this time it continues to entrain ambient fluid, both at the top and sides of the thermals, as well as in cusps of ambient air extending into the mixed layer at the edges of the thermals. This causes the average height of the capping inversion to grow, since on each event some free-flow air is captured and spun up to the turbulence energy level of the mixed layer.

Each impingement may also cause the instantaneous interface between mixed layer and free flow to oscillate; thus a snapshot would show a rather wavy structure. The behavior of thermals during this stage is important

\footnotetext{
1 Permanent affiliation: Meteorology Section, Ris $\varnothing$ National Laboratory, DK-4000, Roskilde, Denmark.

2 The National Center for Atmospheric Research is sponsored by the National Science Foundation.

because of their crucial role in the growth of the ABL through entrainment of free-flow air and the maintenance of the turbulent inversion layer.

A useful basis for an inquiry into the behavior of thermals during this stage is a set of aircraft measurements taken in winter over the East China Sea during the Air Mass Transformation Experiment (AMTEX), where several flights were conducted in the vicinity of a well-defined capping inversion.

To deal with the data it is convenient to have some sort of a thermal model in mind. We envisage an idealized thermal as a vortex ring-like structure with a strong updraft in the middle and relative downdrafts at the outer edges. After impingement, the thermal eventually comes to rest. The internal circulation, however, continues and a strong shear zone enveloping the upper part of the thermal is maintained. Eventually the thermal sinks back into the mixed layer.

Furthermore, a thermal impinging on the capping inversion is assumed to be a coherent entity. If so, it should be possible to detect recurring velocity, temperature and humidity patterns. This is difficult, however, to verify from airplane measurements. A major consideration in analyzing such data is that the nominally level flight path of the airplane cuts in and out of the mixed layer when flying in the vicinity of the corrugated top of the mixed layer. In addition, the airplane is encountering thermals at all stages of their evolution and at random lines of intersection through them. Thus the airplane sees, aperiodically, rising as well as sinking thermals of various scales not connected to the size of the actual thermals. Therefore, looking at the structure of what is believed to be the imprint of a 
thermal requires some kind of conditional analysis; that is, certain sections of a record are categorized according to some criterion, which might to some extent be arbitrary, that distinguishes thermals, preferably also according to maturity. When a suitable ensemble of sections is at hand they might then be investigated for common structure.

These constraints to a proper conditional sampling procedure have to be viewed from the perspective of the amount of data available for analysis. In a first simplified attempt at identifying penetrating thermals we shall ignore most of the above reservations and look at statistics for flight segments without applying any conditional analysis. Thus we are limited to investigating the statistical effects of thermals in the vicinity of the inversion layer, without examining the structure of an individual thermal.

\section{Time series observations}

We use the AMTEX 75 Electra aircraft data for this analysis. A description of the flights is given by Lenschow and Agee (1976). Data from two of the legs are presented in Fig. 1. These legs were selected for further analysis because they so nearly bisect the top of the mixed layer. Measurements of the three wind components are obtained from the Electra air motion sensing system. (Lenschow et al., 1978). Downwardlooking radiation temperature is obtained from a radiation thermometer. Low values of radiation temperature are due to clouds below the aircraft. Temperature is obtained from the standard Rosemount thermometer. Humidity is obtained from a dew-point hygrometer, which has a much slower response time (on the order of several seconds) than the other sensors used for calculating the variables shown in Fig. 1.

Fig. 2 shows a rawinsonde sounding closest, in time and space, to the aircraft flight. The aircraft soundings of potential temperature, humidity and wind, which are obtained from sensors with faster response times, are shown in Figs. 3 and 4. The potential temperature profile and especially the humidity profile show an order-of-magnitude sharper interface than do the profiles measured with the radiosonde. The overlapping curve in the interface region is due to the airplane flying level for some time in this layer (a stretch of $\sim 25 \mathrm{~km}$ ). This gives some indication of the beight variation of the capping inversion, in this case approximately 50-100 $m$. In contrast, the instantaneous thickness of the inversion is on the order of $10 \mathrm{~m}$ or less.

The potential temperature lapse rate between 900 $\mathrm{mb}$, which was the observed cloud-base height, and the inversion base at $850 \mathrm{mb}$ is midway between the dry and the wet adiabatic lapse rate. This is reasonable in

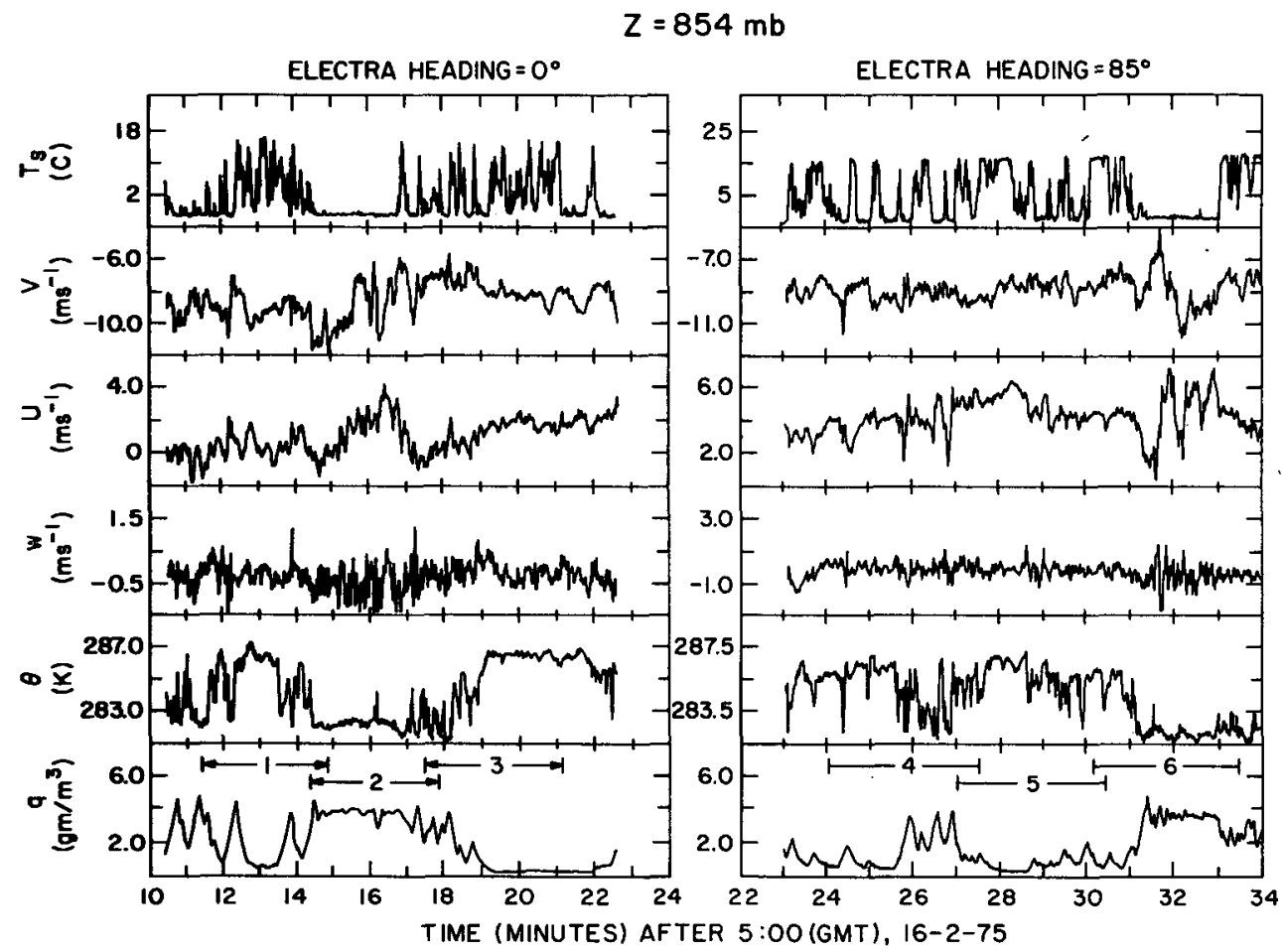

FIG. 1. Time series plots of one sample per second surface temperature, potential temperature, humidity and the three wind components (in geographic coordinates) for two legs on 16 February 1975 at $854 \mathrm{mb}$. The aircraft flew upwind for flight leg on left; crosswind on the right. Near the bottom the six sections used for further detailed analysis are shown. Initial and final coordinates for the flight track are $28^{\circ} 02^{\prime} \mathrm{N}, 124^{\circ} 58^{\prime} \mathrm{E}$ and $28^{\circ} 41^{\prime} \mathrm{N}, 125^{\circ} 45^{\prime} \mathrm{E}$. 


\section{MIXING RATIO $\left(10^{-3}\right)$}

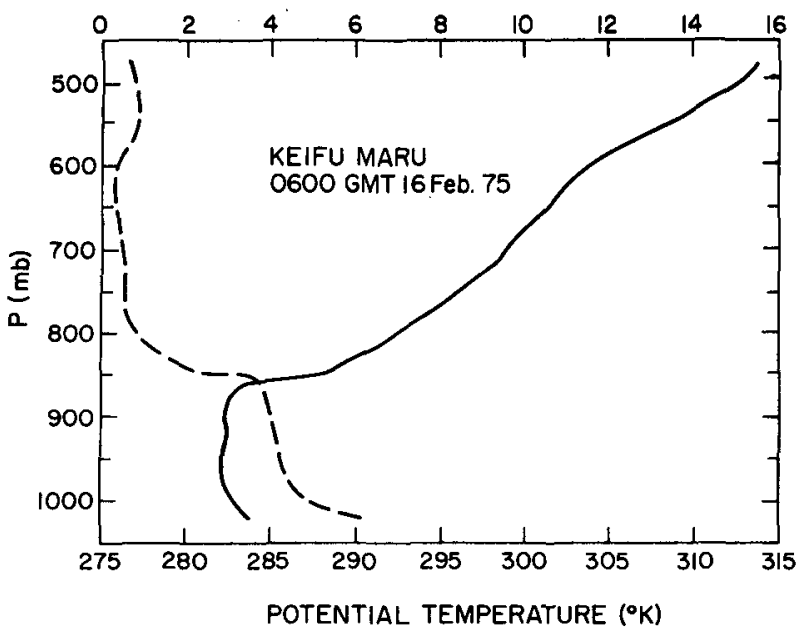

FIG. 2. Rawinsonde sounding from the ship Keifu Maru $\left(28^{\circ} \mathrm{N}\right.$, $125^{\circ} \mathrm{E}$ ). Solid line is potential temperature, dashed line water vapor mixing ratio.

view of the partial cloudiness of this region of the $A B L$. Below $850 \mathrm{mb}$, potential temperature $\theta$ is well mixed down to at least $1010 \mathrm{mb}(\sim 100 \mathrm{~m}$ above the surface). The temperature jump $\Delta \theta$ at the inversion base is as large as $\sim 5 \mathrm{~K}$, and the free flow lapse rate $\gamma$ is $\sim 10^{-2} \mathrm{~K}$ $\mathrm{m}^{-1}$. The jump in water vapor mixing ratio $\Delta q$ is $\sim-3.5 \times 10^{-3}$. Thus, the jump in virtual potential temperature $\Delta \theta_{v}$, which is more relevant for consideration of dry penetrative convection, is $\sim 4.4 \mathrm{~K}$. The height $h$ from the surface to the capping inversion at $850 \mathrm{mb}$, which is the height of the mixed layer, is 1500 $\mathrm{m}$. The mixing ratio $g$ in Fig. 3 appears less well mixed than $\theta$. This is to be expected since moisture is supplied at the surface but dry air is entrained at the mixed

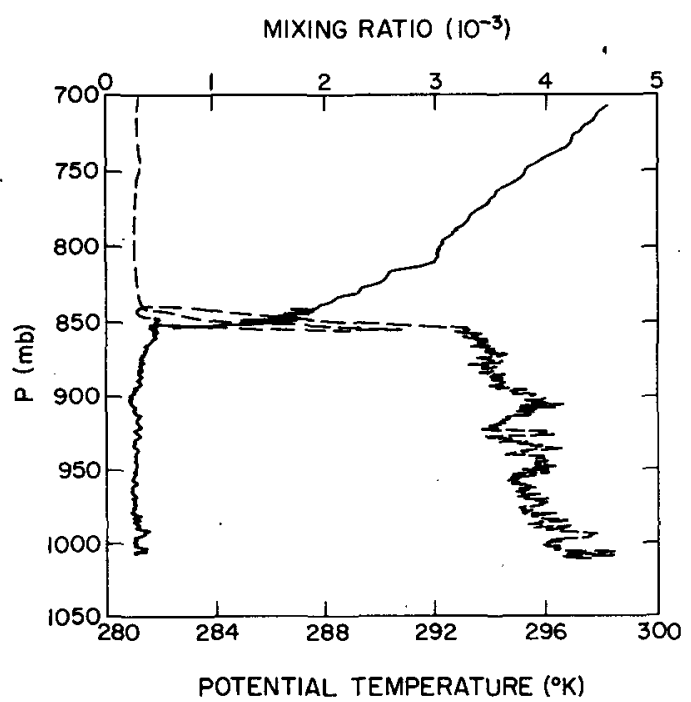

FIG. 3. Aircraft sounding of potential temperature (solid line) and humidity (dashed line). layer top. In contrast, potential temperature is positively enhanced at both these levels (Mahrt, 1976).

Plots of the wind profiles with similar resolution as the above were not very revealing due to the scatter caused by turbulence. With a $30 \mathrm{~s}$ block averaging (this implies horizontal as well as vertical averaging) some structure, however, is apparent in Fig. 4. The northerly component (this is very closely the mean wind) is nearly constant throughout the mixed layer and above, at least to the $700 \mathrm{mb}$ level, with an increase of about $1 \mathrm{~m} \mathrm{~s}^{-1}$ across the top of the mixed layer. The westerly component (or crosswind component) is also constant over the mixed layer. Passing through the capping inversion, however, it increases approximately $3 \mathrm{~m} \mathrm{~s}^{-1}$ and increases approximately linearly with height at a rate of $3 \times 10^{-3} \mathrm{~s}^{-1}$. This is consistent with the baroclinicity in the mixed layer (and in the free atmosphere). Indeed, if the crosswind profile is extrapolated downward it approximately bisects the mixed-layer profile, indicating that the jump results from thorough mixing of that layer.

The first leg of Fig. 1 (0510-0523 GMT), which starts at about $28^{\circ} \mathrm{N}$ and $125^{\circ} \mathrm{E}$, was flown upwind at $850 \mathrm{mb}$ for a distance of about $80 \mathrm{~km}$ north. The second leg (0523-0537) was flown cross wind to the east for about the same distance. These legs were flown at about the average height of the capping inversion, sometimes above and sometimes below the interface, or region of penetration by mixed layer thermals.

As seen from Fig. 1, $80 \mathrm{~km}$ is not substantially larger than the scales of variation in the observed time series. Two major cloud decks, as revealed by the downwardlooking infrared radiometer, occupy some $15 \mathrm{~km}$ each. They are also manifested in the other time series, since the top of the boundary layer is higher in these regions.

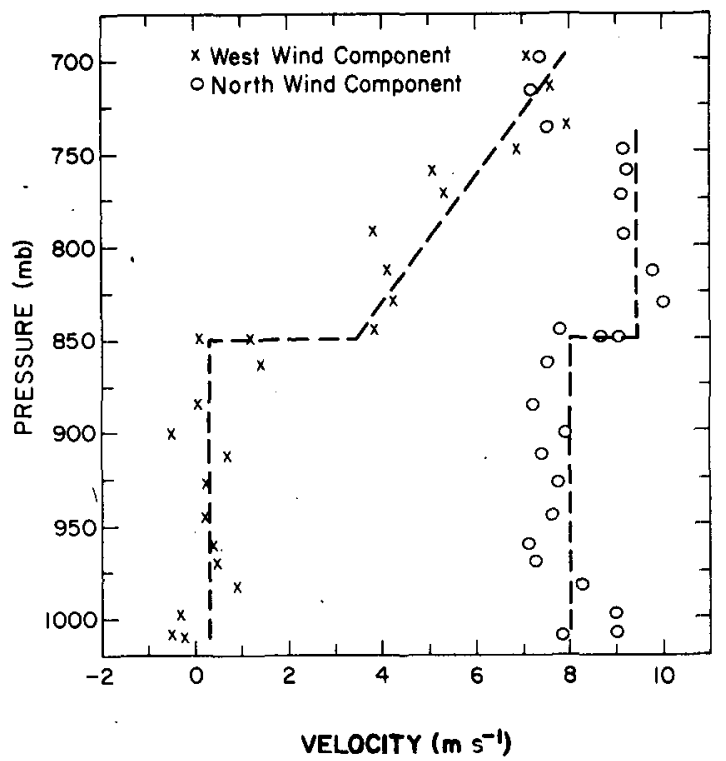

FIG. 4. Aircraft sounding of the horizontal wind components. Each point is a $30 \mathrm{~s}$ average. 
Mixing ratio and potential temperature, for example, have values which are characteristic of the mixed layer with fluctuations much less than when the flight level is alternately above and below the top of the mixed layer.

The $\theta$ and $q$ variations resemble random telegraph signals in that they jump between two preferred states. By comparing with the temperature and humidity soundings, we see that one of the preferred values is representative of the mixed layer and the other of some small height above the mixed layer. The sampling from the two regimes is approximately equipartioned and seems to be a manifestation of the waviness of the interface. A major wavelength is some $20 \mathrm{~km}$ (or 3-4 min of aircraft flight time) which is approximately onefourth of each flight leg. Quasi-periodic variations on smaller scales are also apparent. 'On the basis of the $\theta$ and $q$ traces it is believed that the following three major events took place during each of the two flight legs: 1 ) during the beginning third of leg 1 (flying approximately upwind) the airplane was in the middle of the inversion layer capping the mixed layer; 2 ) in the middle third of that leg, a large cloud was encountered; and 3) in the final third the airplane was mostly above the mixed layer, in the free flow air.

During the first third of leg 2 (crosswind) a number of small clouds were overflown but approximately half of the trace remained in the free flow. The conditions of the next third were largely the same, although the airplane was more frequently in the free flow, and finally in the last third a major cloud formation was again encountered. The anticipated variation of the height of the inversion layer is sketched in Fig. 5.

Over the entire period, mixing ratio and potential temperature are strongly negatively correlated. This observed covariance is expected in clear air on the basis of the observed positive and negative jumps in $\theta$ and $q$, respectively, across the top of the inversion layer. Air drawn down from above the mixed layer along the boundaries of the penetrating thermals by their internal circulation is dry and warm, while air within the thermal is mixed-layer air which is relatively cool and moist. This negative correlation extends even to very small scales, as can be seen in Fig. 6, which is an expanded section of the beginning of leg 1 in Fig. 1. The humidity trace in this figure is obtained from a Lyman-alpha hygrometer, which responds much faster than the dewpoint hygrometer, but is not as accurate for mean humidity measurements. This negative correlation at

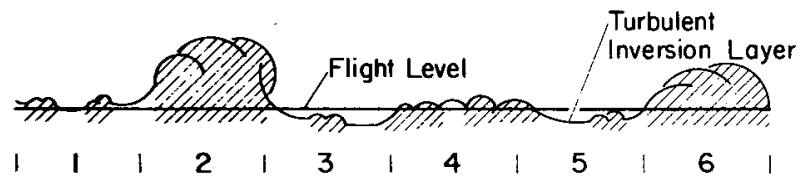

FIG. 5. Schematic diagram of aircraft flight track in the vicinity of the turbulent inversion layer. See Fig. 1 for location of sections $1-6$ on time series plots.

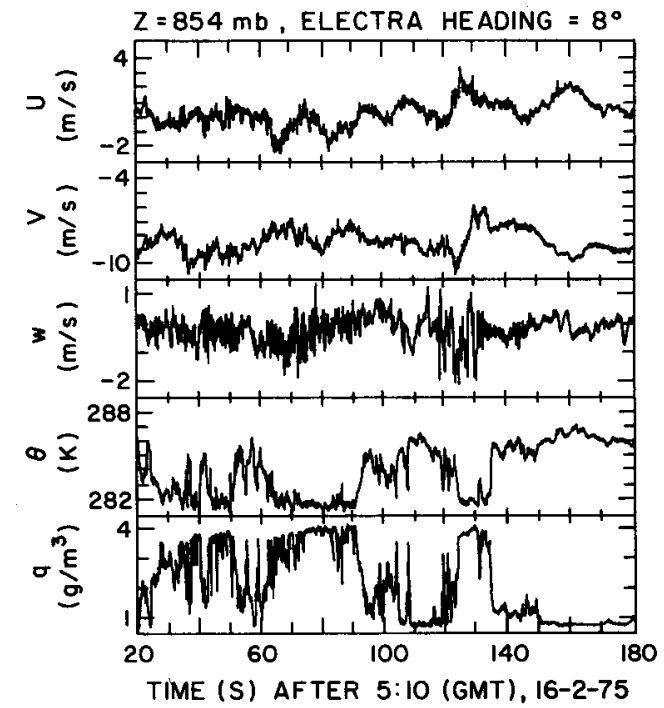

Fig. 6. Expanded portion showing the first $3 \mathrm{~min}$ of leg 1 with data at $20 \mathrm{~s}^{-1}$. The Lyman-alpha hygrometer is used here for humidity, instead of the dew-point hygrometer as in Fig. 1, because of its faster response.

small scales is not surprising, since as Wyngaard et al. (1978) point out, the only effective means for dissipating $\theta-q$ correlation is by molecular dissipation at very small scales (i.e., a few centimeters or less).

This large negative correlation between $\theta$ and $q$ is not expected, however, in a cloud-topped mixed layer. The usual in-cloud assumption, based on the ClausiusClapeyron relationship, is that $\theta$ and $q$ are proportional to each other and therefore positively correlated. Even in the regions predominately cloud covered, however, $\theta$ and $q$ are strongly negatively correlated for the flight legs presented here, which suggests that within clouds near the mixed-layer top there may still be unsaturated parcels of air because of incomplete mixing of the free air with mixed layer air.

\section{Variance and covariance analysis}

We now consider some integral statistical measures of the three wind components, $u$ (positive downwind), $v$ (positive to the left of $u$ ) and $w$ (vertical), as well as $\theta$ and $q$ along the flight path in this interfacial region. For these statistical analyses, the microwave refractometer was used for humidity fluctuations since the Lyman-alpha hygrometer occasionally went off-scale while flying in the very dry air above the mixed layer. When the Lyman-alpha was on-scale, its output agreed very well with humidity obtained from the refractometer. At high frequencies, the refractometer output contains more electronic noise than the Lyman-alpha hygrometer; its effect on the statistics, however, is negligible. Wyngaard et al. (1978) present further details of the temperature and humidity fluctuation measurements.

Results of this analysis, as well as subsequent spectral 
analysis, are more easily interpreted if the time series can be assumed stationary. This is not true for either of the two legs discussed. Major variations occur on scales of the same order of magnitude as the record length. Therefore, some precautions have to be taken. On the other hand, these variations are of sufficiently large scale $(\sim 20 \mathrm{~km})$ that they are presumably not of direct importance for the turbulent entrainment process, but rather a manifestation of mesoscale convective activity, perhaps combined with mesoscale waves. From the standpoint of analyzing the turbulence in these layers, such variations are rather to be viewed as variations of the level of the flight path with respect to the interfacial region. The actual height of the aircraft above the surface remained relatively constant, with a standard deviation over both flight legs of about $2.3 \mathrm{~m}$.

Each of the two flight legs was cut into three slightly overlapping sections of $\sim 20 \mathrm{~km}$ length for more detailed statistical analysis. These sections coincide, by and large, to regions dominated by a particular process as discussed previously and shown schematically in Fig. 5. Each section was detrended separately to reduce the effect of large-scale variations. Values of the standard deviations calculated with respect to the average of each of the six sections are listed in Table 1.

The standard deviations of the horizontal velocity components show a rather systematic stratification: in the two free flow cases they are $\sim 0.5 \mathrm{~m} \mathrm{~s}^{-1}$, in the two cloud penetration cases they are larger than $1 \mathrm{~m} \mathrm{~s}^{-1}$, and in the two remaining cases they are $\sim 0.7 \mathrm{~m} \mathrm{~s}^{-1}$. This is also reflected in the standard deviation of the $u^{\prime} w^{\prime}$ and $v^{\prime} w w^{\prime}$ time series. The mean values of these signals, which added vectorially and multiplied by the density is the stress, vary less obviously for the different cases. The standard deviation of the vertical velocity is also distinctly larger for the two sections primarily in cloud.

As noted earlier in Fig. 4, the observed vertical wind shear across the top of the mixed layer is small along the direction of the wind, but jumps by about $3 \mathrm{~m} \mathrm{~s}^{-1}$ from the west in the crosswind direction. Thus in Table 1 we would expect $\overline{u^{\prime} w^{\prime}}$ to be small and $\overline{v^{\prime} w^{\prime}}$ to be negative because of entrainment of free-flow air. Instead, the values are all too small and scattered to make a reasonable determination of the mean stress in this region.

Values of $\sigma_{\theta}$ and $\sigma_{q}$ are largest in the sections that are closest to being equally above and below the mixed layer top. These values are about ten and four times the mid-level minimum values of $\sigma_{\theta}$ and $\sigma_{q}$, respectively, which is a manifestation of the large jumps in temperature and humidity across the top of the mixed layer. The mid-level minimum (e.g., Kaimal et al., 1976) results from production of $\theta$ and $q$ variance occurring both near the surface and near the top of the mixed layer because of relatively large values of their mean vertical gradients, but negligible production in the middle since the gradients there are small.

It should be pointed out, however, that measurements of $\theta$ may be affected by water phase changes during flights in cloud. If the thermometer were getting wet in cloud, the measured temperature would be cooler than the ambient temperature, as discussed by Lenschow and Pennell (1974). No obvious indication of wetting, however, was observed in the measured temperature. Similarly, if evaporation occurs upstream from the humidity measurement, the measured $q$ may be greater than the actual value. Again, no indication of this was observed in the measured humidity. As a further check on the humidity measurements, humidity obtained from the microwave refractometer, which was used here, was compared to humidity measurement from the Lyman-alpha hygrometer. They were found to agree well with each other.

For comparison with the short sections, statistics are presented in Table 1 for the entire length of each leg. Almost no variance of $w$ is lost in the process of dividing the leg into sections. For $u, v, \theta$ and $q$, however, the average variances are from 20 to $100 \%$ less than those for the entire legs, indicating that the segmenting has

TABLE 1. Statistics for each of the sections of the flight legs at the top of the mixed layer. $\bar{V}$ is the mean wind speed and $\phi$ the wind direction. Other quantities are defined in the text. Average values are averages of the three sections, the total is the entire leg considered as a whole.

\begin{tabular}{|c|c|c|c|c|c|c|c|c|c|c|c|}
\hline & \multirow[b]{2}{*}{1} & \multicolumn{3}{|c|}{$\leftarrow$ Leg I $\left(0^{\circ}\right.$ heading $) \rightarrow$} & \multirow[b]{2}{*}{ Total } & \multicolumn{5}{|c|}{$\leftarrow$ Leg II $\left(85^{\circ}\right.$ heading $) \rightarrow$} & \multirow[b]{2}{*}{ Units } \\
\hline & & 2 & 3 & Average & & 4 & 5 & 6 & Average & Total & \\
\hline$\sigma_{w}$ & 0.41 & 0.59 & 0.35 & 0.45 & 0.46 & 0.47 & 0.39 & 0.70 & 0.52 & 0.53 & $\mathrm{~m} \mathrm{~s}^{-1}$ \\
\hline$\sigma_{u}$ & 0.83 & 1.11 & 0.45 & 0.80 & 1.10 & 0.61 & 0.50 & 1.19 & 0.77 & 0.88 & $\mathrm{~m} \mathrm{~s}^{-1}$ \\
\hline$\sigma_{v}$ & 0.80 & 1.38 & 0.52 & 0.90 & 0.99 & 0.77 & 0.56 & 1.32 & 0.88 & 1.08 & $\mathrm{~m} \mathrm{~s}^{-1}$ \\
\hline$\sigma_{\theta}$ & 1.60 & 0.63 & 0.95 & 1.06 & 1.79 & 1.34 & 0.95 & 1.05 & 1.11 & 1.41 & $\mathbf{K}$ \\
\hline$\sigma_{q}$ & 0.96 & 0.71 & 0.64 & 0.77 & 1.28 & 0.82 & 0.47 & 0.85 & 0.71 & 0.88 & $\mathrm{~g} \mathrm{~m}^{-3}$ \\
\hline$\overline{u^{\prime} w^{\prime}}$ & -0.04 & 0.09 & 0.01 & 0.02 & -0.02 & 0.03 & -0.02 & 0.06 & 0.02 & 0.02 & $\mathrm{~m}^{2} \mathrm{~s}^{-2}$ \\
\hline$\overline{v^{\prime} w^{\prime}}$ & 0.00 & -0.04 & -0.01 & -0.02 & -0.03 & 0.02 & 0.02 & 0.03 & 0.02 & 0.05 & $\mathrm{~m}^{2} \mathrm{~s}^{-2}$ \\
\hline$\sigma_{u^{\prime} w^{\prime}}$ & 0.49 & 0.77 & 0.23 & 0.50 & 0.62 & 0.33 & 0.20 & 1.30 & 0.61 & 0.84 & $\mathrm{~m}^{2} \mathrm{~s}^{-2}$ \\
\hline$\sigma_{v}^{u^{\prime} w^{\prime}} w^{\prime}$ & 0.44 & 0.86 & 0.25 & 0.52 & 0.56 & 0.53 & 0.23 & 1.04 & 0.60 & 0.72 & $\mathrm{~m}^{2} \mathrm{~s}^{-2}$ \\
\hline $\bar{V}$ & 10.62 & 10.70 & 10.10 & 10.46 & 10.46 & 9.66 & 9.47 & 9.36 & 9.49 & 9.49 & $\mathrm{~m} \mathrm{~s}^{-1}$ \\
\hline$\phi$ & 357 & 356 & 357 & 357 & 357 & 352 & 349 & 351 & 350 & 350 & deg \\
\hline
\end{tabular}


removed variance. This means that, in contrast to $w$, these quantities have significant variance at the low wavenumbers.

\section{Spectral analysis}

Spectral and cospectral densities were calculated for the time series discussed previously using a fast Fourier transform algorithm. Because of the short record length $(205 \mathrm{~s}$ or $\sim 20 \mathrm{~km})$ scales greater than several kilometers are not included. However, the larger scales are presumably not important for the entrainment process as such, but merely modulate the smaller scale convective elements which are important for entrainment.

We should also keep in mind that spectral analysis decomposes the time series into a set of discrete Fourier modes which is not a very satisfactory representation of a set of thermals of varying size and spacing which are encountered by the airplane along random lines of intersection. Therefore, we cannot expect to see a sharp peak in the spectrum at some wavelength proportional to an average thermal diameter. Rather, we would hope that the spectra have a broad peak, perhaps over as much as an octave or more, at a wavelength that is characteristic of twice the thermal diameter. We also note that the spacing between thermals seems to be similar in length to the thermal diameter.

Before commenting on the spectra in the entrainment region, we discuss characteristics of spectra at lower levels. In the surface layer the spectrum of $k S_{w}(k)$, where $k$ is wavenumber and $S_{w}(k)$ the spectral density of $w$, is observed to obey classical Monin-Obukhov similarity. For $z / L<-1$, where $L$ is the Obukhov length, free-convection behavior is expected. Indeed,

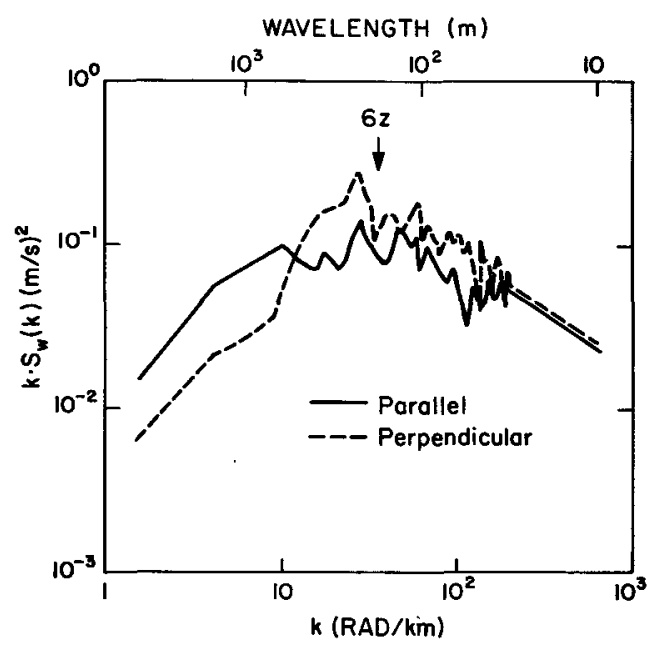

Fig. 7. Vertical velocity spectra calculated from $205 \mathrm{~s}$ sections from cross wind and along wind flight legs during the period 0645:20 to 0657:10 GMT at a height of $z=35 \mathrm{~m}$. Values of the relevant parameters are $u_{*}=0.35 \mathrm{~m} \mathrm{~s}^{-1}, L=-40 \mathrm{~m}$ and $h=1500 \mathrm{~m}$. Spectra in this and in Figs. 8, 10,11 and 12 are calculated for 4096 points sampled at $20 \mathrm{~s}^{-1}$ and averaged to 256 spectral estimates, with about 30 degrees of freedom. The high-wavenumber end of the spectra have been smoothed by eye.

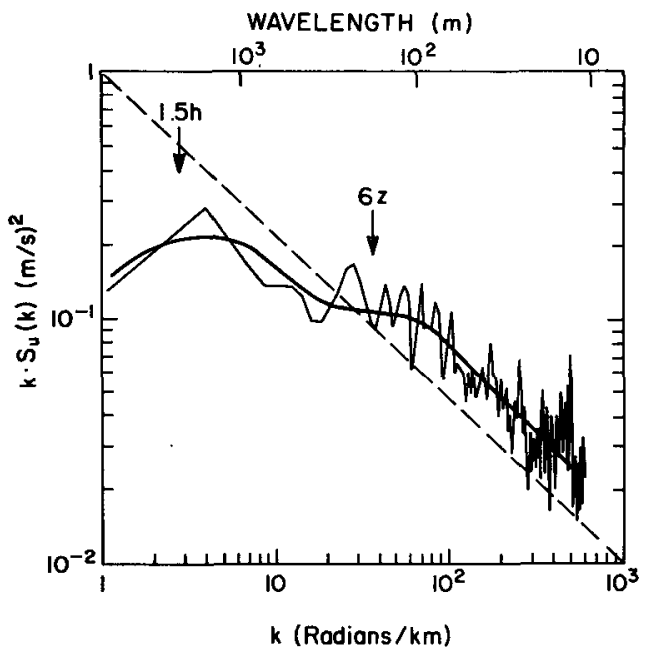

FIG. 8. Spectrum of along wind (u) component of horizontal velocity measured along a cross wind flight leg for the same period as in Fig. 7.

we find that this is the case (Fig. 7). The peak scale $\lambda_{m w}$ of the logarithmic wavenumber spectrum of the vertical velocity component agrees with the limiting value of $\sim 6 z$ found in Kansas as well as in the above-mentioned Minnesota analysis (Kaimal et al., 1972, 1976). We find, however, that the crosswind $w$ spectrum is considerably more peaked than the alongwind spectrum and has less amplitude at long wavelengths. This may be due to elongation of eddies along the direction of the wind.

The spectra of the horizontal velocity components, however, scale with the height of the mixed layer (Kaimal et al., 1976). The present data confirm their findings that the wavelengths of spectral maxima in an unstable boundary layer, $\lambda_{m u}=\lambda_{m v}=1.5 h$, where $h$ is the height of the boundary layer (Fig. 8). For more

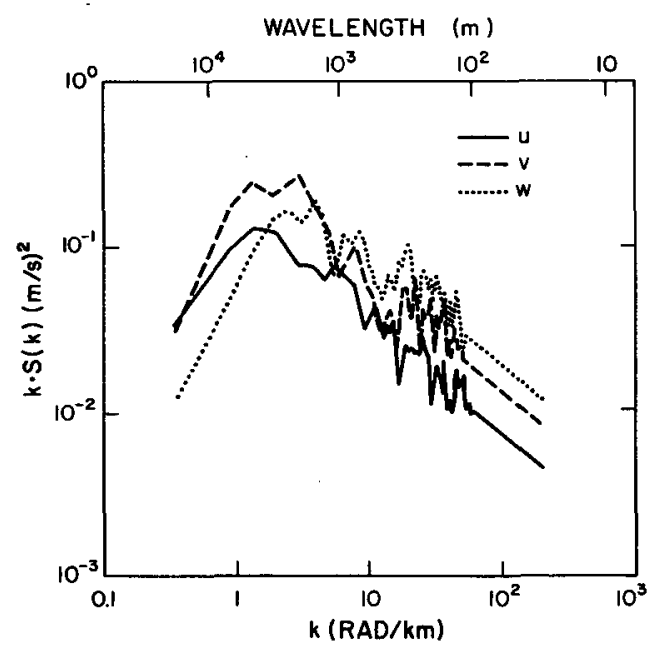

Fig. 9. Velocity spectra measured in the alongwind direction at $900 \mathrm{mb}(z / h \approx 0.7)$ for the period 0549:01 to 0559:00 GMT. Spectra are calculated for 4096 points sampled at $5 \mathrm{~s}^{-1}$ and averaged to 256 spectral estimates. Values greater than $k \approx 60$ have been approximated by a straight line. 
neutral conditions, LeMone and Pennell (1976) have observed spectral peaks at 2-3 $h$. Since the spectra reported by. Kaimal et al. were measured at a fixed location, they applied the Taylor hypothesis to convert to wavenumbers, while the present spectra are essentially directly measured as wavenumber spectra since the aircraft speed is an order of magnitude greater than the mean wind speed. The agreement lends validity to the application of Taylor's hypothesis to the velocity components at these wave lengths. Even such details as the relatively flat "intermediate range" in $k S_{u}(k)$, as found in the unstable Kansas data as well as in the Minnesota data, are confirmed.

In the middle of the mixed layer we find (again in agreement with the Minnesota analysis) that $\lambda_{m}$ of the three velocity components converge. At $z / h \approx 0.7$ the $w$ spectrum is nearly identical to the $u$ and $v$ spectra. This is illustrated in Fig. 9 where $\lambda_{m w} \approx 2 \mathrm{~km}$, which agrees reasonably well with the estimate of Kaimal $e t a l$. (1976) of $1.5 \mathrm{~h}$. The value of $\lambda_{m u}$ does have a tendency, however, to be slightly larger than $\lambda_{m v}$ and $\lambda_{m v}$. Furthermore, the $v$ and $w$ spectral peaks seem again to be narrower in the crosswind directions.
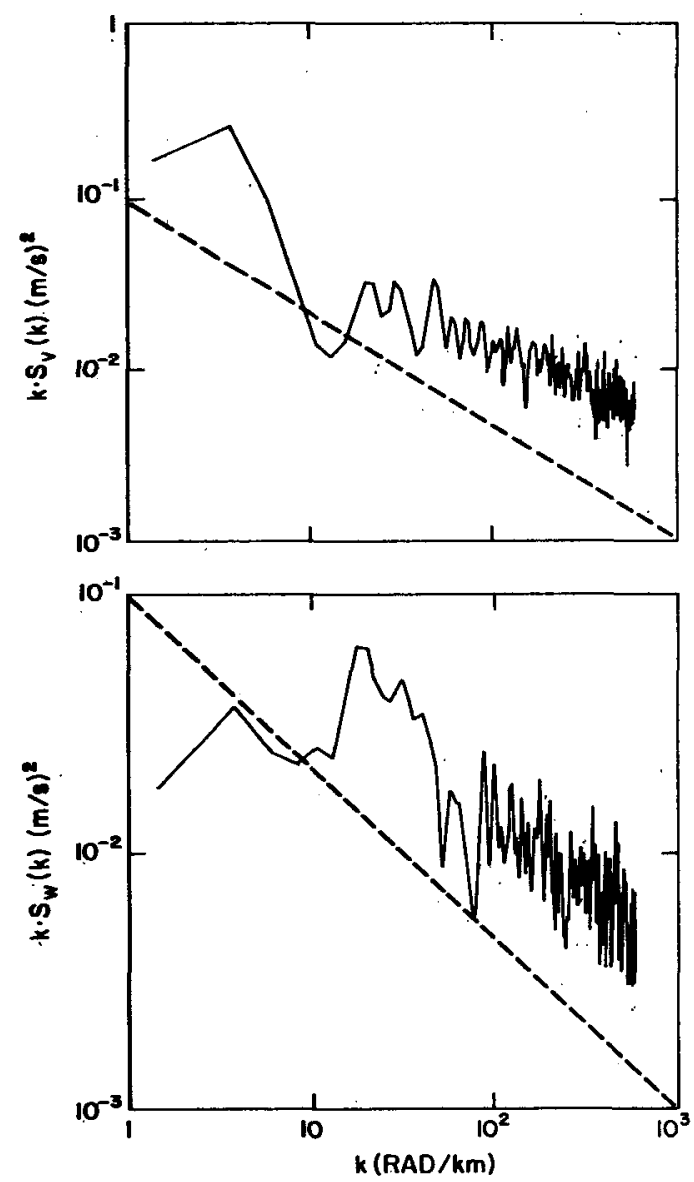

FIG. 10. Spectra of the crosswind and vertical wind components, potential temperature and humidity measured on an alongwind flight path at the turbulent inversion layer $(854 \mathrm{mb})$ from 0511:25 to 0514:49 GMT. The dashed lines have a slope of $-\frac{2}{3}$.
At the top of the mixed layer, the entire $w$ spectrum has been attenuated by approximately a factor of 2 compared to the middle of the mixed layer. Spectra from the first $20 \mathrm{~km}$ section discussed previously are shown in Fig. 10. These spectra have, in general, two separate maxima: 1) the lower wavenumber peak $2 \pi / \lambda_{1}$ corresponds to the abovementioned scale of $1.5 h$ and 2) the other peak is at a scale $\lambda_{2}=200-300 \mathrm{~m}$. While $\lambda_{1}$ seems to correspond to eddy sizes of the order of the boundary layer height, $\lambda_{2}$ seems to be related to some characteristic size of penetrating thermals. At wavelengths shorter than $\lambda_{3} \approx 100 \mathrm{~m}$, corresponding to $k=50 \mathrm{rad} \mathrm{km}^{-1}$, the logarithmic velocity spectra decrease as $\sim k^{-3}$. Some of the temperature spectra, however, have a slope closer to -1 . This is not surprising, since the logarithmic spectrum of the observed step changes in temperature can be expected to have a slope of -1 .

Fig. 11 is an example of the cospectrum of $w$ and $\theta$. A large positive contribution to the covariance is found at wavelengths $>600 \mathrm{~m}$. At shorter wavelengths the cospectrum fluctuates around zero. A wavelength of $600 \mathrm{~m}$ corresponds to the gap between the $\lambda_{1}$ and $\lambda_{2}$
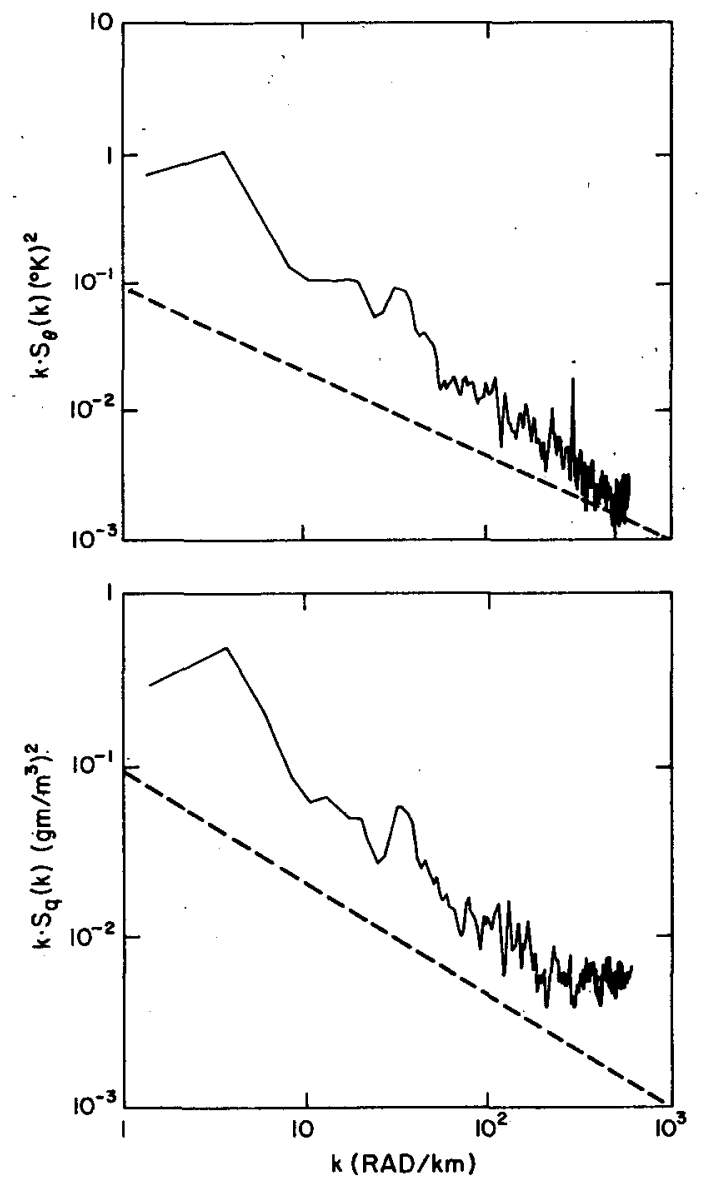
scales in the $k S_{w}(k)$ spectra. Thus the $w$ variance contained in the $\lambda_{2}$ peak is not correlated with $\theta$. The peak of the cospectrum, on the other hand, coincides with the $\lambda_{1}$ scale $(1.5 h)$. The positive covariance, which was found on all six sections, is somewhat surprising, since the temperature flux is expected to be negative near the mixed-layer top due to downward moving entrained free-flow air which is warmer than mixed-layer air. However, Deardorff (1976) shows that in stratocumulus-topped mixed layers the temperature flux may be positive even to within $50 \mathrm{~m}$ of the mixed-layer top. Another possibility is that the measured values may not be representative of the overall area averaged flux due to insufficient sample length or a biased sample. Finally, if some wetting of the thermometer occurred or if salt contamination caused cold spikes (Schmitt et al., 1978) preferentially in downward moving air, an erroneous positive temperature flux would result. No obvious indication of either wetting or salt contamination, however, was observed in the time series of temperature.

Individual $w$ spectra for the $20 \mathrm{~km}$ sections are shown in Fig. 12. At small wavenumbers (long wavelengths) they converge to a common value. Variations in the variance from case to case are accounted for by variable spectral levels in the high-wavenumber region. The local spectral maximum at $\lambda_{2}$ seems to be of greatly varying magnitude compared to the principal peak at $\lambda_{1}$-from considerably larger than the $\lambda_{1}$ maximum (as in case 2, a cloud case) to virtually nonexistent (as in case 5, a free flow case). This description implies that a high turbulence intensity case has a smaller overall $\lambda_{m}$ scale than a low intensity case.

To investigate more fully the possibility of two distinct scales, we computed spectra for each of the two entire legs $(\sim 80 \mathrm{~km}$ each). Each full length $w$ spectrum is similar to what would be expected from averaging

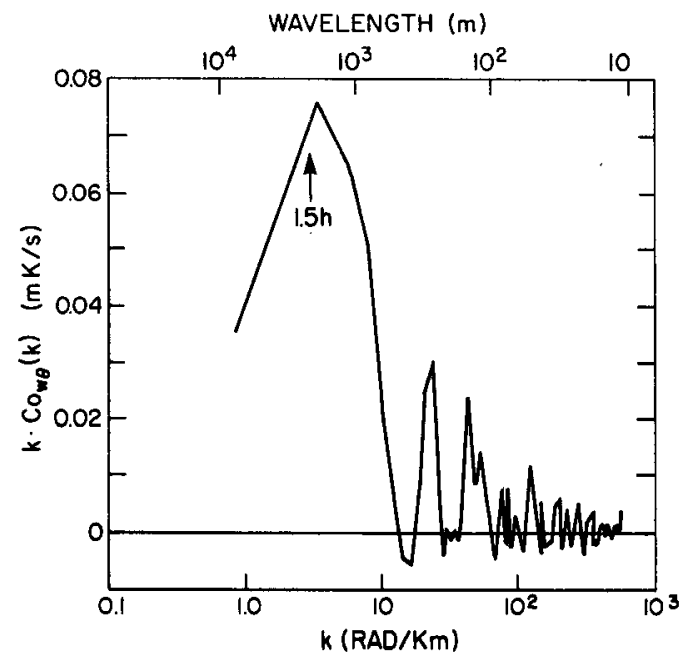

FIG. 11. Cospectrum of vertical velocity and potential temperature for section 4 (0524:01-0527:25) measured crosswind at the turbulent inversion layer $(854 \mathrm{mb})$.

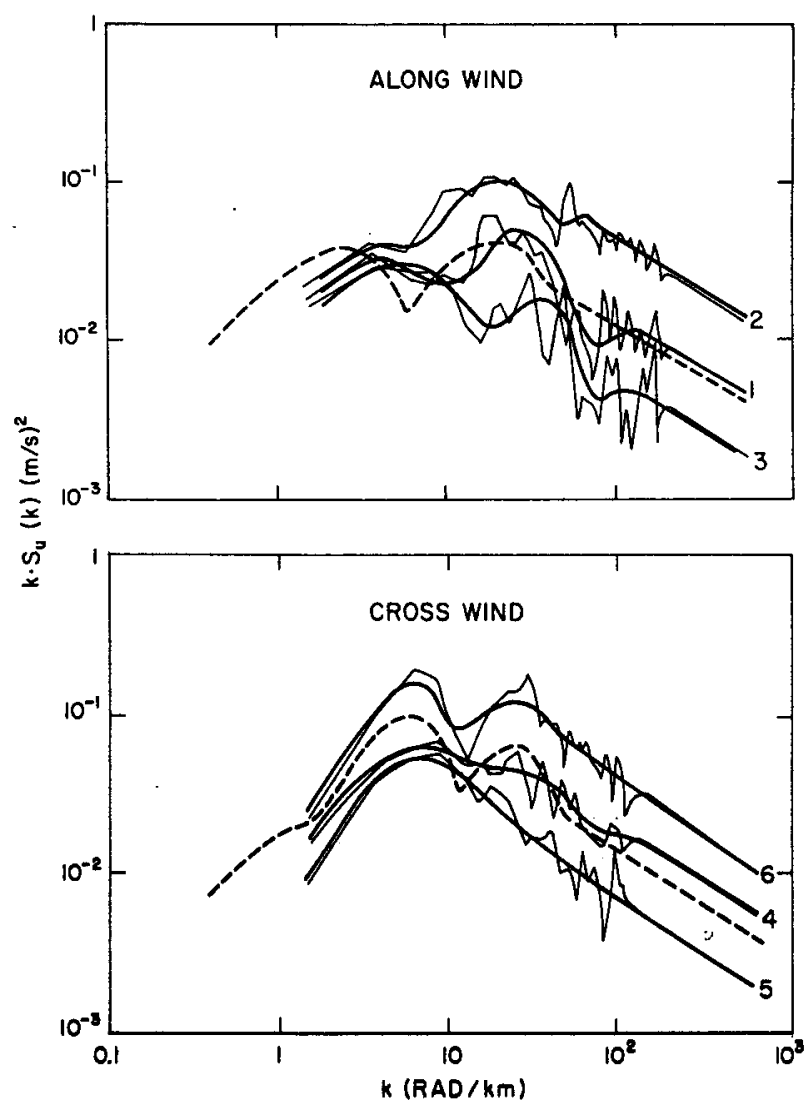

FIG. 12. Vertical velocity spectra for each of the six sections shown in Fig. 1 (thin lines). Thick solid lines are smoothed by eye. Dashed lines are smoothed spectra calculated for the entire flight leg, using 4096 samples at five samples per second. At the highwavenumber end of the curve, spectral values are approximated by a straight line.

the three separate cases of each leg except that the gap between the two scales is accentuated in the full length spectra.

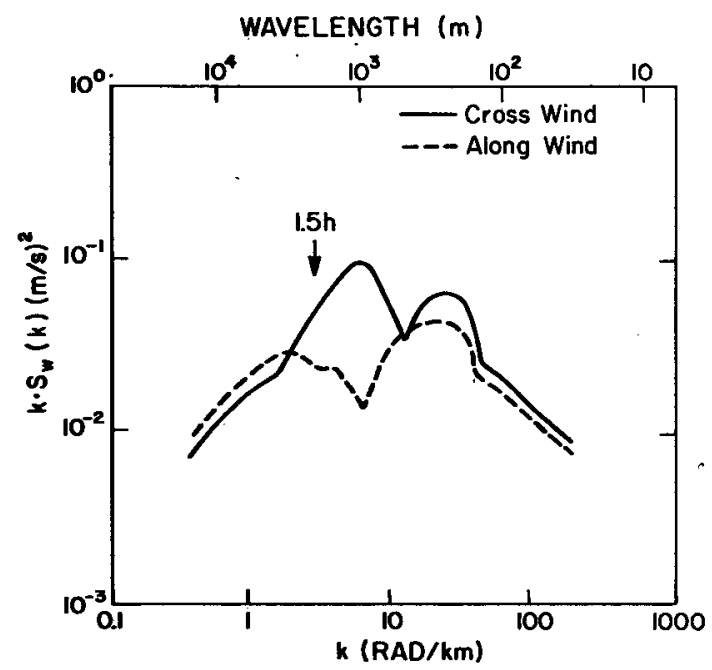

FIG. 13. The two spectra from Fig. 11 corresponding to full-length flight legs plotted together. 


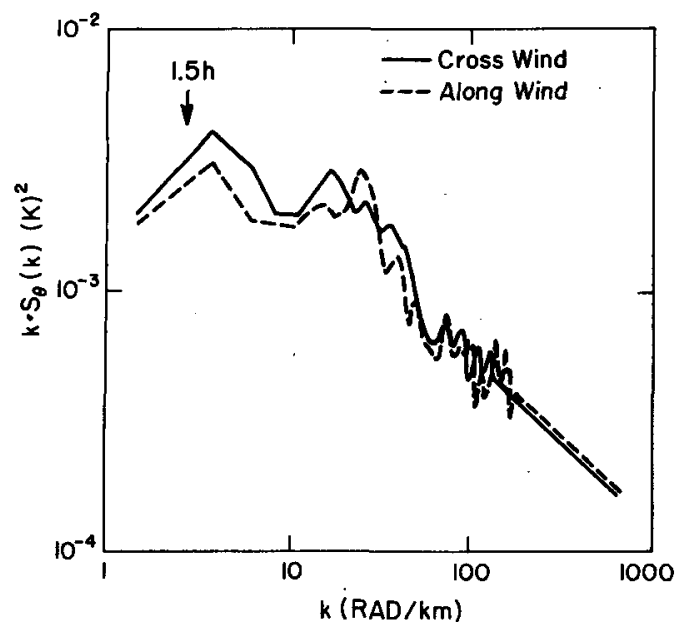

FIG. 14. Potential temperature spectra at $900 \mathrm{mb}(z / h \approx 0.7)$. The alongwind spectrum is from 0549:01 to $0555: 30$, the crosswind spectrum from $0538: 11$ to $0547: 45$.

Fig. 13 shows that the spectral level at small wavenumbers is nearly the same in the two cases, as is the size and position of the peak at $\lambda_{2}$, as well as the energy level in the cascade range. The differences in size of $\lambda_{1}$ and the magnitude of the spectral density at this wavelength depending on orientation relative to the mean wind is interesting. It appears that eddies characteristic of this scale are elongated structures. This feature is not evident from the $w$ spectra in the mixed layer or in the surface layer, except that the crosswind spectra appear more peaked. However, some of the $\theta$ spectra from the surface layer (not shown) can be distinguished according to alongwind and crosswind directions by the position of the logarithmic maximum.

If we now look specifically for double-peaked spectral shapes in the middle of the mixed layer, we find a definite double peak in the $\theta$ spectra. The $\lambda_{2}$ peak is very narrow but occurs consistently at the $200-300 \mathrm{~m}$ scale as shown, for example, in Fig. 14. In this figure, the alongwind spectrum has about 60 degrees of freedom and the crosswind about 90 degrees of freedom. Assuming the spectral density estimates follow a chisquare distribution, both peaks have a very high significance, since each spectral estimate has an $80 \%$ probability of being within about $20 \%$ of its ensemble average.

While extending the length of the time series in the entrainment region to the length of the entire flight legs does not significantly affect the average $w$ spectrum, the spectra of the horizontal velocity components are altered. The maxima of the logarithmic spectra of $u$ and $v$ shift to a longer wavelength: $\lambda_{m} \sim 6 \mathrm{~km}$, which is $2.4 h$ as compared to $1.5 h$ in the mixed layer (Fig. 15). This longer wavelength is more in agreement with the results of LeMone and Pennell (1976) for a more nearly neutral boundary layer. This behavior was expected from the integral statistics analysis, where it was found that the

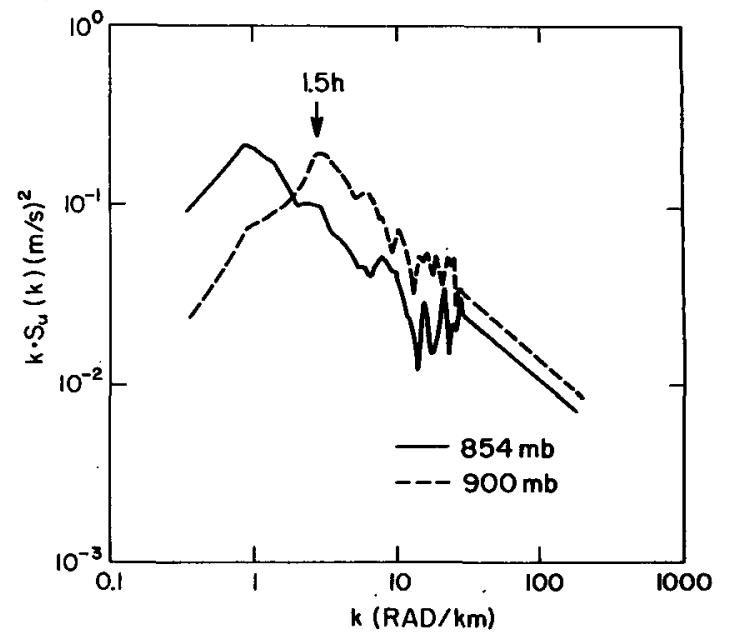

FIG. 15. Spectra of alongwind component measured on a crosswind flight path at the turbulent inversion layer $(854 \mathrm{mb})$ from 0523 to 0534 and in the mixed layer $(900 \mathrm{mb})$ from 0538 to 0548 .

variance was considerably larger over a full leg compared to the average of the results from the shorter cases. This additional variance occurs at the lowest wavenumbers, whereas, on scales smaller than $1.5 h$, the spectra are the same as in the mixed layer or slightly attenuated, as were the $w$ spectra.

\section{Conclusions}

On the basis of a detailed analysis of two aircraft flight legs of $80 \mathrm{~km}$ length each, flown at the top of a partially cloud-covered convective boundary layer, some aspects of penetrative convection have been illustrated and examined. Penetrating thermals, which are identified by spectral peaks in vertical velocity and temperature at a wavelength of $200-300 \mathrm{~m}$, are separable in size and structure from larger scale mixed-layer eddies which scale with boundary layer height $h$. This double-peaked spectral characteristic can be identified in temperature spectra at lower levels as well. Horizontal velocity spectra at the top of the mixed layer do not have a spectral peak associated with penetrating thermals. They do have a peak at $\sim 2.4 h$, which agrees with the results of LeMone and Pennell (1976), compared to $1.5 h$ in the middle of the mixed layer. The mid-layer results agree well with the tethered balloon measurements reported by Kaimal et al. (1976).

Over the entire time period, mixing ratio and temperature are strongly negatively correlated out to very small wavelengths, which is expected in clear air but not expected in the cloud-covered regions. Vertical velocity and temperature are positively correlated out to a wavelength of $600 \mathrm{~m}$, which is in the gap between the two spectral peaks, with negligible correlation at shorter wavelengths.

Although some interesting details have emerged from this analysis, further analysis would be useful to verify 
how general these observations are under different sets of conditions and with different observational strategies. An analysis of covariances of $\theta$ and $q$, $w$ and $\theta$, and $w$ and $q$ in this interfacial region, which is now underway, may be able to provide further details on the process of penetrative convection.

Acknowledgments. Most of the painstaking data processing for this study was done by Y. Pann. P. Stephens performed some additional calculations and D. Howard typed the manuscript. We also acknowledge the excellent support of the NCAR Research Aviation Facility and the Computer Facility Data Management Group in gathering and processing the original AMTEX data set. L. Mahrt contributed many useful ideas and suggestions. A portion of this research was supported by National Science Foundation Grant ATM76-81908.

\section{REFERENCES}

Deardorff, J. W., 1976: On the entrainment rate of a stratocumulus-topped mixed layer. Quart. J. Roy. Meteor. Soc., 102, 563-582.
Kaimal, J. C., J. C. Wyngaard, Y. Izumi and O. R. Coté, 1972: Spectral characteristics of surface layer turbulence. Quart. J. Roy. Meteor. Soc., 98, 563-589.

—- D. D. Haugen, O. R. Coté, Y. Izumi, S. J. Caughey and C. J. Readings, 1976: Turbulence structure in the convective boundary layer. $J$. Atmos. Sci., 33, 2152-2169.

LeMone, M. A., and W. T. Pennell, 1976: The relationship of trade wind cumulus distribution to subcloud layer fluxes and structure. Mon. Wea. Rev., 104, 524-539.

Lenschow, D. H. and W. T. Pennell, 1974: On the measurements on in-cloud and wet-bulb temperatures from an aircraft. Mon. Wea. Rev., 102, 447-454.

-, and E. M. Agee, 1976: Preliminary results from the Air Mass Transformation Experiment (AMTEX). Bull. Amer. Meteor. Soc., 44, 1346-1455.

- C, C. R. Cullian, R. B. Friesen and E. B. Brown, 1978: The status of air motion measurements on NCAR aircraft. Preprints 4th Symp. Meteorological Observations and Instrumentation, Denver, Amer. Meteor. Soc., 433-438.

Mahrt, L., 1976: Mixed layer moisture structure. Mon. Wea. Rev., 104, 1403-1407.

Schmitt, K. F., C. A. Friehe and C. H. Gibson, 1978: Humidity sensitivity of atmospheric temperature sensors by salt contamination, J. Phys. Oceanogr., 8, 151-161.

Wyngaard, J. C., W. T. Pennell, D. H. Lenschow and M. A. LeMone, 1978: The temperature-humidity covariance budget in the convective boundary layer. $J$. Atmos. Sci., 35, 47-58. 